\title{
Flow Around Flapping Flexible Flat Plate Wings
}

\author{
Diego Campos ${ }^{1}$ and Lawrence Ukeiley ${ }^{2}$ \\ University of Florida, Shalimar, FL, 32579 \\ Luis Bernal ${ }^{3}$ \\ University of Michigan, Ann Arbor, MI, 48109,
}

\begin{abstract}
This study investigates the unsteady flow phenomena generated from plunging wings with varying flexibility. The experiments were conducted in a wind tunnel with forward flight conditions at a chord based Reynolds number of 5300. The wings are fabricated out of homogeneous isotropic materials with $\Pi_{1}$ values of 55 and 1702. The conditions of these experiments are matched to previous studies in a water channel to investigate the requirement of dynamic similarity utilizing the flexible wing similarity parameter $\left(\Pi_{1}\right)$. Stereo Particle Image Velocimetry is used to measure the phase-averaged flow around the wings in several spanwise planes along the wing. The flow data provides insight into the evolution of vortices formed at the leading edge of the wing. This data is also used to estimate the forces created by the wings using a momentum balance method. The measurements showed that large deflections at the tip produced strong leading edge vortices. However, when the tip-root lag is greater than $180^{\circ}$, the effects are adverse resulting in no leading edge vortex development. In order to understand wing flexibility further, additional experiments were performed using a laser Doppler Vibrometer to understand the modal properties for each wing with varying flexibility parameter. These studies are designed to gain further insight into future experiments that attempt to control the amount of deformation on the wing by altering the ratio of plunging (excitation) to the natural frequency.
\end{abstract}

\section{Introduction}

$I^{N}$ $\mathrm{N}$ recent years there has been an interest in studying and understanding natural flyers to incorporate some of their features into small engineered flying systems. In order to achieve this goal, a better understanding of the unsteady fluid phenomena generated over various flapping kinematics which are utilized by insects and birds to achieve the aerodynamic forces necessary for flight is desired. The mechanisms that govern the generation of aerodynamic forces are associated with the formation and shedding of vortices into the flow. Therefore, an understanding of the fluid-structure interactions between the vortex dynamics and the structural properties of the wing is of great importance ${ }^{1}$.

Flapping wing flight is typically characterized by low flight speeds which results in a low chord based Reynolds numbers (Re). In this regime, the dynamics of vortex structures and resulting aerodynamic characteristics have been shown to be affected by varying the Reynolds numbers. Studies by Shyy, et al ${ }^{1}$ and Tang et $\mathrm{al}^{2}$ demonstrated that for realistic biological wing body configurations, Re was the dominant parameter dictating the transient development of vortex structures. They also showed the ability of the Reynolds number to alter the flow symmetry between the upstroke and downstroke. A Re of $\mathrm{O}\left(10^{3}\right)$ showed flow asymmetry between strokes, as it is experienced in the current study. Rausch et $\mathrm{al}^{3}$ presented the effects of spanwise flexibility on the aerodynamics of plunging, isotropic wings. They showed that the introduction of flexibility caused greater three-dimensional flow towards the tip of the wing. It was also shown that the development of a larger leading edge vortex was the result of an increase in plunge induced angle of attack. The formation and evolution of these vortical structures and their

\footnotetext{
${ }^{1}$ Graduate Research Assistant, Department of Mechanical and Aerospace Engineering, University of Florida, AIAA Student Member

2 Associate Professor, Department of Mechanical and Aerospace Engineering, University of Florida, AIAA Associate Fellow

${ }^{3}$ Associate Professor, Department of Aerospace Engineering, University of Michigan, AIAA Member

1

American Institute of Aeronautics and Astronautics
} 
effects on the force production has been documented in previous studies ${ }^{1,4-6}$. This study will focus on wing flexibility and the resulting fluid-structure interactions. This interaction is of interest because previous studies have shown that flexibility is important in bird and insect flight ${ }^{7}$, and essential in fish swimming ${ }^{8}$. Furthermore, recent studies have shown increased thrust coefficients and efficiencies ${ }^{9,10}$ for intermediate stiffness at constant Strouhal numbers (St). To better understand the effects of the wing stiffness, it is important to have non-dimensional scaling parameters relating wing material characteristics to the free stream conditions. One of the kinematic parameters of importance is the Strouhal number, which describes the relative influence of the free stream velocity to the flapping frequency and amplitude. The Strouhal number typical of natural, flapping wing, flyers ranges from 0.2 to $0.4^{11}$. A recent study by Heathcote et al ${ }^{10}$ showed an increase to propulsive efficiency for $\mathrm{St}>0.2$. They also stated that the reason for decreases in efficiency for Strouhal numbers not between 0.2 and 0.4 may be a result of increased flow separation at higher $\mathrm{St}$ and a transition to drag at lower $\mathrm{St}^{9}$. Several scaling parameters for fluid-structure interaction of flapping flight have been provided in the review by Shyy, et al ${ }^{6}$. This study will concentrate on varying the $\Pi_{1}$ which is the ratio of elastic and aerodynamic forces, and is defined as

$$
\Pi_{1}=\frac{D}{\rho_{\infty} U_{\infty}^{2} c^{3}}
$$

where $\rho_{\infty}$ is the air density $U_{\infty}$ is the free stream velocity, and $\mathrm{c}$ is the root chord. The flat plate bending stiffness (D) is defined as

$$
D=\frac{E t^{3}}{12\left(1-\gamma^{2}\right)}
$$

where $\mathrm{E}$ is the modulus of elasticity, $\mathrm{t}$ is the thickness, and $\gamma$ is Poisson's Ratio.

The wing kinematics and Reynolds number will be fixed in this study to investigate the effect of wing flexibility on the resultant fluid flow. This will not only allow for the development of an understanding of the structural stiffness but also be comparable to water tunnel studies reported in Rausch et $\mathrm{al}^{3}$. Comparison of these studies will provide insight into the effects of density ratio between the wing and fluid surrounding it. Recent investigations have focused on understanding the effect of chordwise and spanwise flexibility for isotropic wings on force generation and efficiency ${ }^{12}$. These investigations showed the importance of the added mass force in the natural flight regime. A base for the study of the added mass effects were provided from scaling relationships for aerodynamic forces and wing deflection derived as a function of the density ratio, natural and flapping frequency ratio, reduced frequency and flapping amplitude ${ }^{13,14}$.

Recent reviews are aimed at comprehending the connection between excitation frequency and the structural properties (mass, density, bending and twisting modes) of natural flyers. Particular interest is placed upon the ratio of flapping to natural frequency and its relationship to the maximum propulsive force and maximum efficiency. These investigations showed that for a flat plate model, the maximum force was generated at a frequency slightly lower than the natural frequency of the wing ${ }^{13,15,16}$. Similarly, numerical results utilizing the lattice Boltzmann method showed that the maximum propulsive force occurred at a frequency ratio of 0.95 . It was shown that the maximum force increased when inertial effects of the wing became more dominant than the fluid inertia ${ }^{17}$. The propulsive efficiency of a flapping wing in a hovering environment was investigated numerically by Vanella ${ }^{18}$. He showed that the ratio for maximum efficiency is significantly lower than the ratio for maximum force, at 0.33 , and that it was caused by the enhancement of the wake capture mechanism. This is a consequence of stronger streamwise flow during the upstroke induced from a strong vortex at the trailing edge. Studies carried out using a self-propelled, simplified insect model also showed that optimal propulsive efficiency is obtained at only a fraction of the natural frequency ${ }^{13,15}$.

The objective of this study is to understand the effect of varying the wing stiffness parameter $\left(\Pi_{1}\right)$ on the flow and forces as it is compared to water tunnel experiments reported by Rausch et $\mathrm{al}^{3}$. Additionally, by using the wing stiffness parameter to characterize the flexibility and structural properties of the wings, a relationship can be identified between the ratios of excitation frequency to natural frequency on the wing deflections.

\section{Experiments}

The following section provides a description of the facilities and the experimental equipment used. The section also includes a discussion of the models tested, and the relevant experimental parameters. 


\section{II.A Aerodynamic Characterization Facility}

The experiments presented in this paper have been conducted in the Aerodynamic Characterization Facility (ACF) at the University of Florida's Research and Engineering Education Facility. This wind tunnel is an open jet open return facility specifically designed for low Reynolds number experiments. The tunnel entrance is comprised of a flow conditioning system along with an 8:1 area contraction ratio. Exiting the contraction through a $1.07 \mathrm{~m} \mathrm{x}$ $1.07 \mathrm{~m}$ opening, the flow enters the open jet test section with an axial length of 3.05 meters. The wind tunnel has the capability of achieving free stream velocities between approximately 0.5 and $22 \mathrm{~m} / \mathrm{s}$. The flow has a uniform core of at least $60 \%$ of the cross sectional area at the midpoint in the test section. The vertical and horizontal centerline mean freestream velocities vary within $0.03 \mathrm{~m} / \mathrm{s}$ for a mean freestream velocity of $2 \mathrm{~m} / \mathrm{s}$. Additionally, experiments showed that turbulence intensities were less than $0.07 \%$ for a free stream velocity around $2 \mathrm{~m} / \mathrm{s}$ which is the free stream velocity being used in these experiments. Further details of the flow quality and a more extensive description of the ACF can be found in Albertani et al ${ }^{19}$.

\section{II.B Plunging Device}

A plunging mechanism was designed and built to perform a plunging kinematic motion for the experiments presented in this paper. Figure 1 is a photograph of the plunging mechanism. The device is driven by a Maxon EC $1615 \mathrm{~W}$ Brushless DC motor with hall sensors. It contains a planetary gear head with a 57/13 reduction ratio. This results in a maximum input speed of $8000 \mathrm{rpm}$. The motors' MR M type encoder provides a pulse signal with 4096 pulses per revolution, which allows the EPOS2 24/5 Positioning Controller to determine the position of the motor with a precision of $1 / 1024$ th revolution or $0.35^{\circ}$. The controller obtains position and velocity feedback from the encoder and regulates the motor. The output speed range for the motor shaft is $0-30 \mathrm{~Hz}$, and is controlled to within $1 \%$ of the desired speed. The plunging mechanism works by transforming the rotation output from the motor into a reciprocating motion which slides a linear bearing. The root of the wing is attached to a structure that moves with the bearing, thus having a sinusoidal plunging motion at the desired amplitude.

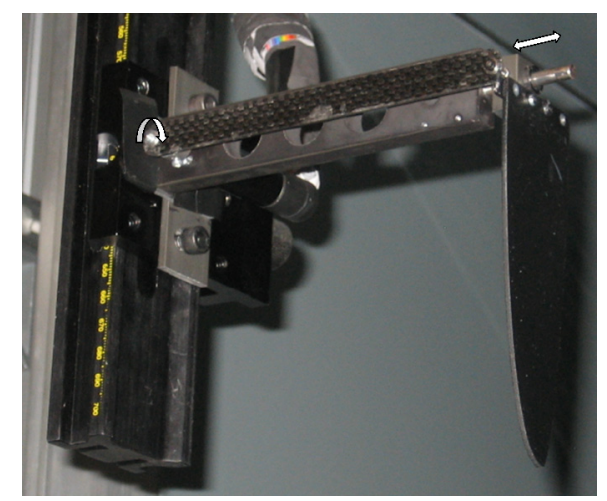

Figure 1. Plunging device with wing attached.

\section{II.B.1 Motion Analysis}

The plunging mechanism is designed to have a repeatable sinusoidal motion, defined by

$$
h(t)=h_{a} \cos (2 \pi f t)
$$

where $h_{a}$ is the plunging amplitude of the motion and $f$ is the plunging frequency. The accuracy of the motion was verified using a high speed camera to track the wing's root. Images were acquired at a rate of $500 \mathrm{~Hz}$ and an in house Matlab script was written to interpret the root locations within the images. Figure 2 presents the commanded motion (tracking sinusoid) against the measured location of the wing root (bearing position).The motion deviates slightly at the extremes as the wing root comes to a complete stop. The loads are also significantly greater at this position in the kinematic motion. After performing a spectral analysis on the acquired locations, it was determined that the desired motion is achieved to within $0.1 \mathrm{~Hz}$. 


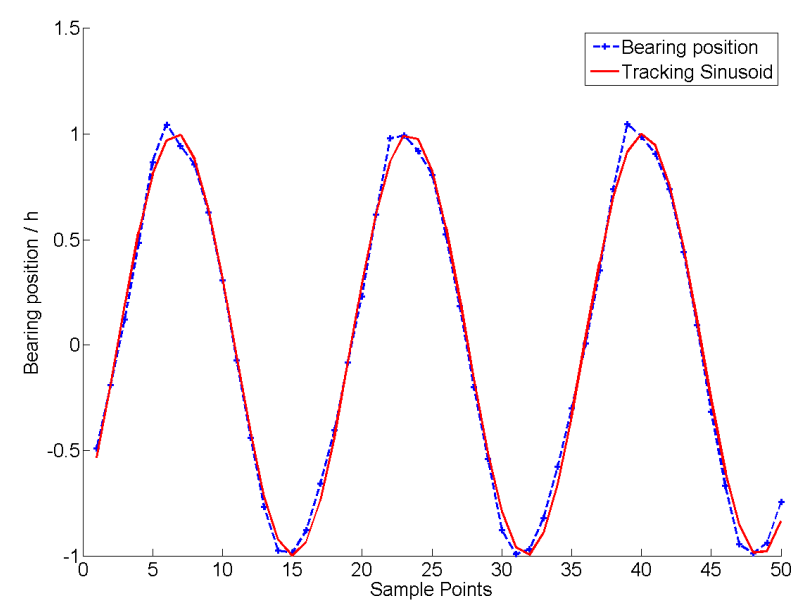

Figure 2. Plunging Device Sinusoidal Motion Analysis

\section{II.B.2 Synchronization with PIV}

The relative wing position for each PIV snapshot is determined by sampling the encoder position at each instant the PIV cameras are triggered. A NI-DAQ 6220 is used to sample the motor encoder signal on two channels and the PIV trigger signal on the third. A C based computer software is utilized to process the encoder output and track the motion. The program samples when it receives the first PIV trigger signal, which is then used to synchronize with the motor position.

\section{II.C. Wing}

The wings utilized in these experiments had a Zimmerman planform which is defined as two ellipses meeting at the quarter chord point. Specific dimensions are shown in Figure 3(a) resulting in an aspect ratio of 7.65, defined by

$$
A R=\frac{4 b^{2}}{S}
$$

where $\mathrm{S}$ is the surface area of the wing, and $\mathrm{b}$ is its semispan. The wings are manufactured from 3 homogeneous isotropic materials: low density polyethylene (LDPE), high density polyethylene (HDPE), and Ultra-High Molecular Weight polyethylene (UHMW). This provides various $\Pi_{1}$ values that span three orders of magnitude ranging from $10^{1}$ to $10^{3}$. Two different wing configurations are used and shown in Figure 3. For the first wing configuration presented in Figure 3(a), the wing is attached to the plunging device such that it is fully supported along the entire root, thereby constraining twist. The second wing configuration is presented in Figure 3(b). This configuration varies from the first by only being attached along the first third of the wing root such that twist is unconstrained.

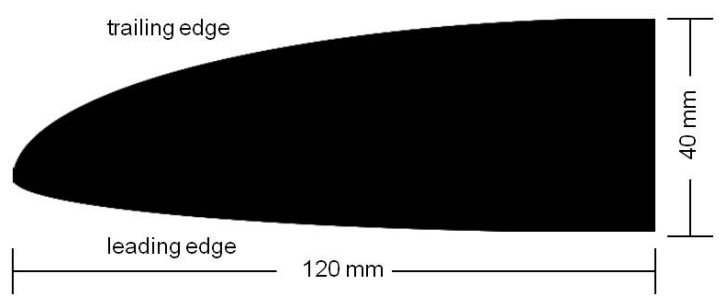

(a)

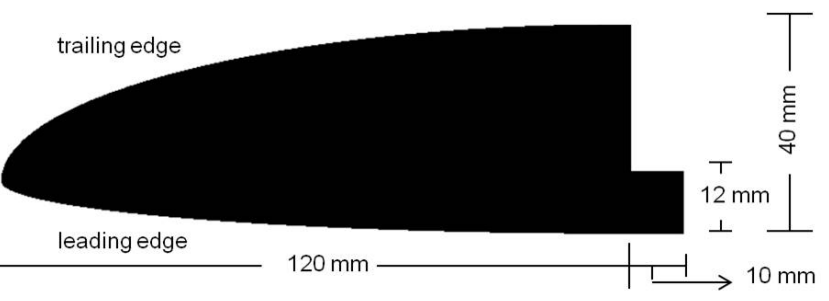

(b)

Figure 3. Zimmerman Planform wings. (a) wing used for the PIV experiments, (b) wing with unconstrained twist 


\section{II.D. Particle Image Velocimetry}

A LaVision Particle Image Velocimetry (PIV) system controlled by LaVision's imaging software Davis 7.2 was used to measure the flow field around the plunging wings. The system consists of a Litron Nano L 135-15 laser which is used to create a pair of $532 \mathrm{~nm}$ pulses into light sheets in the vertical spanwise direction. Two Imager ProX-4M cameras were used to capture the light scattered from particles at oblique viewing angles. The cameras are equipped with a 14 bit dynamic range CCD array of 2048 x 2048 pixel resolution. The cameras were coupled with Sigma EX $105 \mathrm{~mm}$ lenses with an f-number of 5.6. This resulted in an average resolution of 19 pixels per $\mathrm{mm}$. The flow was seeded by a LaVision DS Aerosol Generator which used olive oil as the seeding material and generated particles on the order of $1 \mu \mathrm{m}$.

Stereoscopic PIV was used to acquire three component velocity data. The raw PIV images were processed using Davis 7.2 PIV software. The software uses a multi-pass, cross-correlation algorithm. The first pass consisted of an interrogation window of 64 pixels x 64 pixels with a 50\% overlap. The two subsequent passes utilized a window size of $32 \times 32$ pixels with a $50 \%$ overlap. The estimated displacement from the each previous pass was used to displace the raw image such that the same particle group would be correlated for smaller interrogation window sizes.

For each wing, measurements were acquired at 6 spanwise locations ranging from $60 \mathrm{~mm}$ to $108 \mathrm{~mm}(50 \%$ to $90 \%$ span) where $0 \%$ and $100 \%$ are the wing root and tip respectively. A schematic of the spanwise locations is shown in Figure 4. At each spanwise location, 5000 velocity fields were acquired and divided into 50 phases over the full plunging cycle. This resulted in approximately 120-145 velocity fields which are used to create the mean flow field at each phase in the plunging motion.

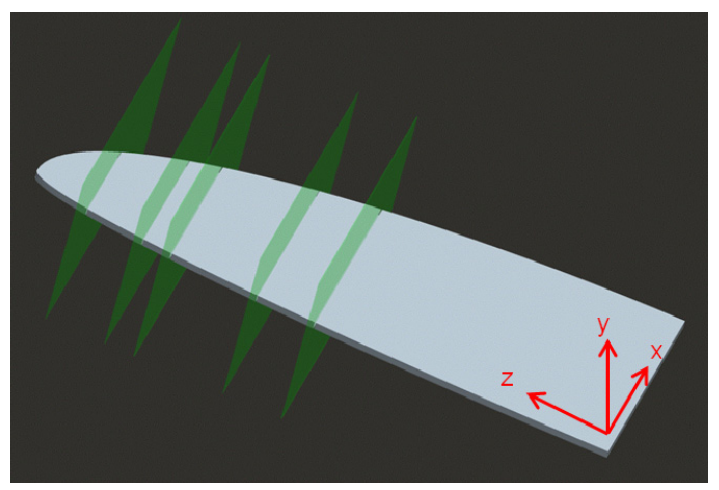

Figure 4. PIV planes and coordinate system.

\section{II.E. Laser Doppler Vibrometry}

A modal analysis was performed on the wings to determine their damped natural frequency. The structural response of the wings was measured using a noninvasive Polytec PSV-400 laser Doppler Vibrometry (LDV). The LDV system consists of a PSV-I-400 laser head with a He-Ne laser that emits a linearly polarized $633 \mathrm{~nm}$ beam, a OFV-5000 controller, and a PSV-E-401 Junction box for synchronizing all the hardware. A Ling Dynamic Systems V201/3-PA electrodynamic shaker with an attached load cell was used for the excitation. Each wing was rigidly attached to the shaker, as can be seen in Figure 5. For all experiments, the shaker setup is placed on a vibration isolated optical table exposed to ambient air. Therefore, the results include aerodynamic damping and would differ slightly from the true natural frequencies which would need to be determined in a vacuum.

\section{II.E.1 Structural Deformation Analysis Methods}

The Polytec software was used to obtain the LDV output and the load cell data to calculate the frequency response structure of the wings studied. To perform these measurements, the laser is first focused and centered on the middle of the grid spacing, such that the location error is minimized at 6 points on the wing surface. A grid of approximately 60 points was created for each wing. The laser was refocused at each point to obtain the best signal to noise ratio. The shaker provided a burst chirp excitation to the wing and the response of the wing was captured by the LDV laser head. The data was acquired over the course of 5 burst chirps at each point on the grid, and then averaged to obtain the frequency spectrum at each point. Another form of excitation utilized was sine sweep excitation. Excitation frequencies were varied from $0-1000 \mathrm{~Hz}$ with sweeps over 6 seconds of length. Ample time between measurements was allowed such that the structure returned to steady state. For each experiment the 
software samples the points until 5 coherent measurements are obtained, discarding those that are not within acceptable error bounds.

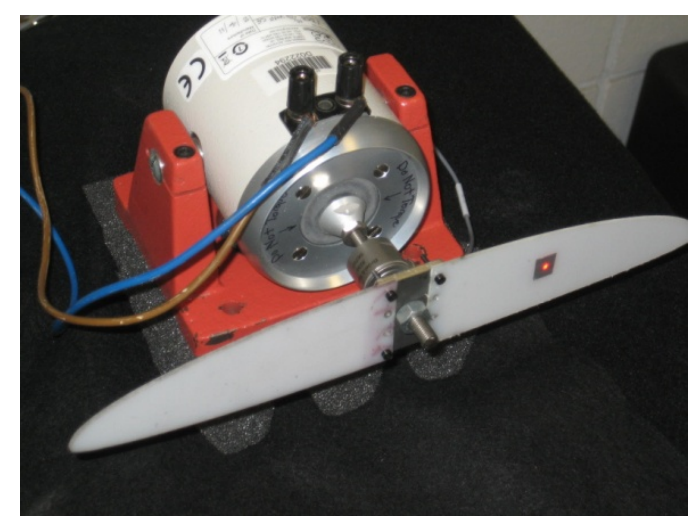

Figure 5. Shaker and Wing Setup.

\section{II.F. Non-Dimensional Parameters}

As stated previously, by fixing the kinematics and the Reynolds number, the effect of the spanwise flexibility on the fluid dynamics of the plunging wings can be investigated. The flexibility is varied by changing the effective stiffness $\left(\Pi_{1}\right)$ over three orders of magnitude. The relevant scaling parameters for the study of plunging flexible wings are provided by Baik ${ }^{20}$, and are obtained through performing a dimensional analysis using the fluid density, flow velocity, and the chord as the basis variables ${ }^{14,21}$. These were also derived by evaluating the kinematics and governing equations for the structure (flat plate) and fluid ${ }^{1,22}$.

The experimental parameters and flow properties are provided in the following tables. Table 1 summarizes the relevant scaling parameters; Table 2 summarizes the kinematics and geometric properties; Table 3 summarizes the material properties, and Table 4 shows the fluid-structure scaling parameter values.

Table 1. Dimensionless kinematic scaling parameters

\begin{tabular}{|c|c|c|c|}
\hline $\mathbf{R e}=\mathrm{c} U_{\infty} / \nu$ & $\mathbf{k}=\pi f c / U_{\infty}$ & $\mathbf{S t}=2 \mathrm{f}_{\mathrm{o}} / U_{\infty}$ & $\mathbf{h}_{\mathbf{o}}=\mathrm{h} / \mathrm{c}$ \\
\hline 5300 & 1.82 & 0.203 & 0.175 \\
\hline
\end{tabular}

Table 2. Kinematic and Geometric Properties

\begin{tabular}{|c|c|c|c|c|}
\hline $\begin{array}{c}\text { Root } \\
\text { Chord }\end{array}$ & Span & Velocity & Frequency & Plunging Amplitude \\
\hline $\boldsymbol{c}(\boldsymbol{m m})$. & $\boldsymbol{b}(\boldsymbol{m m})$. & $\boldsymbol{U}_{\infty}(\boldsymbol{m} / \mathbf{s})$ & $\boldsymbol{f}(\boldsymbol{H z})$ & $\boldsymbol{h}(\mathbf{m m})$. \\
\hline & & & & \\
\hline 40 & 120 & 2.1 & 30 & 7.00 \\
\hline
\end{tabular}

Table 3. Material Properties

\begin{tabular}{|c|c|c|c|c|c|}
\hline LDPE & & HDPE & & UHMW & \\
\hline $\mathrm{E}(\mathrm{GPa})$ & $\begin{array}{c}\text { Poisson's } \\
\text { Ratio }\end{array}$ & $\mathrm{E}(\mathrm{GPa})$ & $\begin{array}{c}\text { Poisson's } \\
\text { Ratio }\end{array}$ & $\mathrm{E}(\mathrm{GPa})$ & $\begin{array}{c}\text { Poisson's } \\
\text { Ratio }\end{array}$ \\
\hline 0.39 & 0.32 & 1.50 & 0.35 & 0.62 & 0.46 \\
\hline
\end{tabular}

Table 4. Dimensionless fluid-structure scaling parameters

\begin{tabular}{|c|c|c|c|c|}
\hline $\begin{array}{c}\prod_{1,1 / 32} \\
\text { LDPE }\end{array}$ & $\begin{array}{l}\prod_{1,1 / 16} \\
\text { LDPE }\end{array}$ & $\begin{array}{c}\prod_{1,1 / 32} \\
\text { HDPE }\end{array}$ & $\begin{array}{c}\prod_{1,1 / 16} \\
\text { HDPE }\end{array}$ & $\begin{array}{c}\prod_{1,1 / 16} \\
\text { UHMW }\end{array}$ \\
\hline 55 & 444 & 213 & 1,702 & 795 \\
\hline
\end{tabular}




\section{Force Estimation}

The phase-averaged forces generated by the plunging wing are computed from the flow field data using the method described in Sällström ${ }^{23,24}$. The control volume spans the extent of the 6 planes where velocity data was acquired, therefore the data has to be significantly interpolated in between the PIV planes to utilize this method. The force is underestimated as the velocity fields only span $40 \%$ of the total wing span.

The method is derived from the incompressible, momentum equation for a Newtonian fluid with the assumption of constant density $(\rho)$ and dynamic viscosity $(\mu)$. The equation governing this can be defined as

$$
\rho \frac{\partial u_{i}}{\partial t}+\rho u_{j} \frac{\partial u_{i}}{\partial x_{j}}=-\frac{\partial p}{\partial x_{i}}+\mu \frac{\partial^{2} u_{i}}{\partial x_{j} \partial x_{j}}+f_{i}
$$

where $p$ is the pressure, $f$ is the force per unit volume, $u_{i}$ is the velocity tensor, and $x_{i}$ is the Cartesian coordinate tensor. Reynolds decomposition is performed on the component, assuming that the flow is periodic. The bar accent denotes average whereas the tilde represents instantaneous fluctuations. Equations 6-8 define the velocity, pressure, and force respectively.

$$
\begin{gathered}
u_{i}=\bar{u}_{i}+\tilde{u}_{i} \\
p_{i}=\bar{p}_{i}+\tilde{p}_{i} \\
f_{i}=\bar{f}_{i}+\tilde{f}_{i}
\end{gathered}
$$

Substituting Equations 6-8 into Equation 5 yields,

$$
\rho\left(\frac{\partial \bar{u}_{i}}{\partial t}+\frac{\partial \tilde{u}_{i}}{\partial t}+\left(\bar{u}_{j}+\tilde{u}_{j}\right) \frac{\partial\left(\bar{u}_{i}+\tilde{u}_{i}\right)}{\partial x_{j}}\right)=-\frac{\partial \bar{p}}{\partial x_{i}}-\frac{\partial \tilde{p}}{\partial x_{i}}+\mu \frac{\partial^{2} \bar{u}_{i}}{\partial x_{j} \partial x_{j}}+\mu \frac{\partial^{2} \tilde{u}_{i}}{\partial x_{j} \partial x_{j}}+\bar{f}_{i}+\tilde{f}_{i}
$$

By taking the average of the equation above, Equation 10 is obtained. The terms are then rewritten using the incompressible, continuity equation, $\frac{\partial u_{i}}{\partial x_{i}}=0$, to obtain the final expression that will be used to solve for relative pressure and force (Equation 11).

$$
\begin{gathered}
\rho\left(\frac{\partial \bar{u}_{i}}{\partial t}+\bar{u}_{j} \frac{\partial \bar{u}_{i}}{\partial x_{j}}+\overline{\tilde{u}_{j} \frac{\partial \tilde{u}_{\imath}}{\partial x_{j}}}\right)=-\frac{\partial \bar{p}}{\partial x_{i}}+\mu \frac{\partial^{2} \bar{u}_{i}}{\partial x_{j} \partial x_{j}}+\bar{f}_{i} \\
\rho\left(\frac{\partial \bar{u}_{i}}{\partial t}+\frac{\partial \overline{u_{\imath} u_{j}}}{\partial x_{j}}\right)=-\frac{\partial \bar{p}}{\partial x_{i}}+\mu \frac{\partial^{2} \tilde{u}_{i}}{\partial x_{j} \partial x_{j}}+\bar{f}_{i}
\end{gathered}
$$

\section{III.A. Pressure Estimation}

The pressure gradient is given by rearranging equation 10 as,

$$
\frac{\partial \bar{p}}{\partial x_{i}}=-\rho\left(\frac{\partial \bar{u}_{i}}{\partial t}+\frac{\partial \overline{u_{\imath} u_{j}}}{\partial x_{j}}\right)+\mu \frac{\partial^{2} \tilde{u}_{i}}{\partial x_{j} \partial x_{j}}
$$

The assumption of no net body forces on the control volume is used. Therefore, all the terms on the right side are a function of the flow velocity and can be determined from PIV data. The pressure gradient is then integrated over the $\mathrm{x}$ and $\mathrm{y}$ directions to obtain the relative pressure with respect to atmospheric pressure across the domain for each phase-averaged velocity.

\section{III.B. Momentum Balance}

Integrating Equation 10 over a control volume (V) yields, 


$$
\iiint_{V} \rho\left(\frac{\partial \bar{u}_{i}}{\partial t}+\frac{\partial \overline{u_{\imath} u_{j}}}{\partial x_{j}}\right) d V=\iiint_{V}\left(-\frac{\partial \bar{p}}{\partial x_{i}}+\mu \frac{\partial^{2} \tilde{u}_{i}}{\partial x_{j} \partial x_{j}}+\bar{f}_{i}\right)
$$

Using the divergence theorem and rearranging for the force results in Equation 14, where the first term represents the forces exerted on the flow by the wing.

$$
\iiint_{V} \bar{f}_{i} d V=\iiint_{V} \rho \frac{\partial \bar{u}_{i}}{\partial t} d V+\iint_{S} \rho \overline{u_{\imath} u_{j}} n_{j} d S+\iiint_{V} \frac{\partial \bar{p}}{\partial x_{i}} d V
$$

Integrating a rectangular volume with bounds $x_{1} \leq x \leq x_{2}, y_{1} \leq y \leq y_{2}$, and $z_{1} \leq z \leq z_{2}$, defines the force along the $\mathrm{y}$ and $\mathrm{x}$ axis exerted on the flow as shown in Equation 15 and 16. For this case only the relative pressure is required as any offset to the pressure will cancel.

$$
\begin{gathered}
\bar{F}_{y}=\int_{x_{1}}^{x_{2}} \int_{y_{1}}^{y_{2}} \int_{z_{1}}^{z_{2}} \rho \frac{\partial \bar{v}}{\partial t} d x d y d z+\left[\int_{y_{1}}^{y_{2}} \int_{z_{1}}^{z_{2}}\left(\rho \overline{u v}-\mu \frac{\partial \bar{v}}{\partial x}\right) d y d z\right]_{x_{1}}^{x_{2}} \\
+\left[\int_{x_{1}}^{x_{2}} \int_{z_{1}}^{z_{2}}\left(\bar{p}+\rho \overline{v^{2}}-\mu \frac{\partial \bar{v}}{\partial y}\right) d x d z\right]_{y_{1}}^{y_{2}} \\
+\left[\int_{x_{1}}^{x_{2}} \int_{y_{1}}^{y_{2}}\left(\rho \overline{v w}-\mu \frac{\partial \bar{v}}{\partial z}\right) d x d y\right]_{z_{1}}^{z_{2}} \\
\bar{F}_{x}=\int_{x_{1}}^{x_{2}} \int_{y_{1}}^{y_{2}} \int_{z_{1}}^{z_{2}} \rho \frac{\partial \bar{u}}{\partial t} d x d y d z+\left[\int_{y_{1}}^{y_{2}} \int_{z_{1}}^{z_{2}}\left(\bar{p}+\rho \overline{u^{2}}-\mu \frac{\partial \bar{u}}{\partial x}\right) d y d z\right]_{x_{1}}^{x_{2}} \\
+\left[\int_{x_{1}}^{x_{2}} \int_{z_{1}}^{z_{2}}\left(\rho \overline{u v}-\mu \frac{\partial \bar{u}}{\partial y}\right) d x d z\right]_{y_{1}}^{y_{2}}+\left[\int_{x_{1}}^{x_{2}} \int_{y_{1}}^{y_{2}}\left(\rho \overline{u w}-\mu \frac{\partial \bar{u}}{\partial z}\right) d x d y\right]_{z_{1}}^{z_{2}}
\end{gathered}
$$

By using the continuity equation, the first term can be written as follows. This will allow for the term to be evaluated using only PIV flow data on the surface of the volume.

$$
\begin{aligned}
\int_{x_{1}}^{x_{2}} \int_{y_{1}}^{y_{2}} \int_{z_{1}}^{z_{2}} \rho \frac{\partial \bar{v}}{\partial t} & d x d y d z \\
& =\rho \frac{\partial}{\partial t}\left(\left.\int_{x_{1}}^{x_{2}} \int_{z_{1}}^{z_{2}} \bar{v}\right|_{y=y_{1}}\left(y_{2}-y_{1}\right) d x d z\right. \\
& \left.-\int_{y_{1}}^{y_{2}} \int_{y_{1}}^{y}\left(\int_{z_{1}}^{z_{2}}[\bar{u}]_{x_{1}}^{x_{2}} d z+\int_{x_{1}}^{x_{2}}[\bar{w}]_{z_{1}}^{z_{2}} d x\right) d \tilde{y} d y\right)
\end{aligned}
$$

\section{Results}

This study builds on past work performed in a water tunnel facility using the same planform wing and the same scaling parameters ${ }^{3,21}$. The PIV measurements reported here are performed on models that were fully supported along the wings root with a $\Pi_{1}$ of 55 (LDPE) and a $\Pi_{1}$ of 1702 (HDPE). Therefore, the results can be directly compared with the rigid and flexible wings studied previously by Rausch et $\mathrm{al}^{3}$. In addition, a structural deformation analysis is performed on root-attached and leading edge attached wings. These wings incorporate four different $\Pi_{1}$ values and are discussed to develop an understanding of the wings' modal properties. 


\section{IV.A. Modal Analysis}

The modal characteristics of the models were measured using the LDV system coupled with shaker excitation. Table 5 presents the results for the first two modes for the 10 wing models. The last column of the table states whether the wings modes were characteristic of any twist deformation. Two of the 10 models used in the modal analysis were utilized for PIV experiments. These two models are highlighted in Table 5. For the remainder of this paper, these two models will be considered M1 and M2 for the HDPE and LDPE model respectively.

Table 6 shows that the two models used for PIV measurements are tested at a ratio of plunging frequency to damped natural frequency significantly different than previous water tunnel experiments. Even though other parameters are matched, the wing loading is significantly different between experiments. This is a consequence of the ratio $\left(\mathrm{f} / \mathrm{f}_{\mathrm{n}}\right)$ driving the nature of the wing deflection. The ratio is more than an order of magnitude greater for both wings in the current study, with the $\mathrm{O}\left(10^{1}\right)$ wing being excited at almost three times its natural frequency. Figure 5 is a contour plot of the first displacement mode for the M1 and M2 wing models. Both wings have increased displacement from the root of the wing to the tip. The LDPE has a displacement almost twice as large as the HDPE wing over the entire wing.

The first two modes for the root-attached wings were the first and second bending mode, and did not showed twist. The wings attached at the leading edge flap showed a damped natural frequency reduction of approximately $30 \%-40 \%$, and showed twist for the second mode.

Table 5. Natural Frequencies based on modal analysis for the 10 wing models tested.

\begin{tabular}{|l|c|c|c|c|c|c|}
\hline Material & Thickness (in.) & $\boldsymbol{\Pi}_{\mathbf{1}}$ & Attachment & Mode 1 (Hz.) & Mode 2 (Hz.) & Twist Mode \\
\hline \hline HDPE & $1 / 16$ & 1702 & Root & 45 & 228 & No \\
\hline & $1 / 32$ & 213 & Root & 20 & 106 & No \\
\hline & $1 / 16$ & 1702 & LE & 30 & 145 & Yes \\
\hline LDPE & $1 / 32$ & 213 & LE & 12.5 & 68 & Yes \\
\hline & $1 / 16$ & 444 & Root & 24 & 130 & No \\
\hline & $1 / 32$ & 55 & Root & 10.5 & 60 & No \\
\hline & $1 / 16$ & 444 & LE & 15 & 82 & Yes \\
\hline UHMW & $1 / 32$ & 55 & LE & 6.8 & 34 & Yes \\
\hline & $1 / 16$ & 795 & Root & 47 & 235 & No \\
\hline
\end{tabular}

Table 6. Ratio of plunging to damped natural frequency for wings studied in two fluid mediums

\begin{tabular}{|c|c|c|}
\hline & Water Tunnel & Wind Tunnel \\
\hline $\boldsymbol{\Pi}_{\mathbf{1}}$ & $\boldsymbol{f} / \boldsymbol{f}_{\boldsymbol{n}}$ & $\boldsymbol{f} / \boldsymbol{f}_{\boldsymbol{n}}$ \\
\hline $\mathrm{O}\left(10^{1}\right)$ & 0.17 & 2.83 \\
\hline $\mathrm{O}\left(10^{3}\right)$ & 0.03 & 0.67 \\
\hline
\end{tabular}
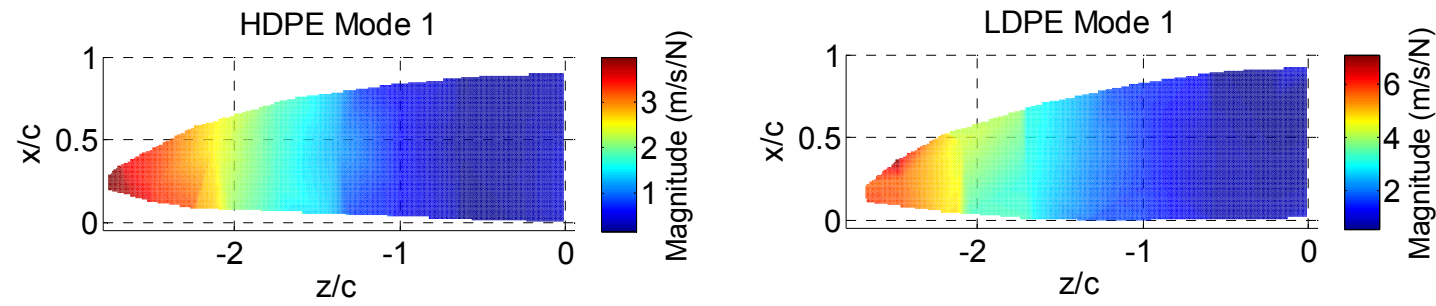

Figure 6. First Mode (Bending) for both wings tested in PIV experiment. 


\section{IV.B. PIV Results}

Details of the phase averaged velocity field characteristics at the $50 \%$ and $75 \%$ spanwise planes for the M1 and M2 models are presented in the following section. These two models are selected such that they can be directly compared with the previous water tunnel studies ${ }^{3}$. The following sections present various contour plots of the flow field around each wing. Due to the laser bloom and wing deformation blocking the field of view, regions of the flow are masked out. These regions correspond to the white regions associated with the figures. Therefore, the near wall effects and the early formation of leading edge vortices cannot be studied from this data set. To clarify the wing position at each phase a black line is plotted to represent the wing's chord location in that plane. This information is achieved from tracking the laser bloom on the PIV images to within approximately $1 \mathrm{~mm}$. All of the flow field results represent a phase averaged field. In these plots the $0^{\circ}$ phase is the beginning of the downstroke while $180^{\circ}$ is the beginning of the upstroke. In addition to plotting the vorticity and velocity fields plots of the Q-criterion ${ }^{25}$ is used to visualize the evolution of the vortical structures in the flow. This criterion identifies vortices as regions with values of Q greater than zero; these representing areas with a larger rotation rate that strain rate. Q criterion is defined by,

$$
Q=\frac{1}{2}\left(\Omega_{i j} \Omega_{j i}-S_{i j} S_{i j}\right)
$$

where $S_{i j}$ and $\Omega_{i j}$ are the symmetric and anti-symmetric component of $\frac{\partial u_{i}}{\partial x_{j}}$, respectively.

\section{IV.B.1 M1 Model}

At the beginning of the downstroke, presented in Figure 7, the M1 wing is at its largest upward deflection. Concurrently, a trailing edge, counter-clockwise vortex is starting to form on top of the wing at the $50 \%$ span location. Vortex development at the leading edge cannot be seen because the wing deflection blocks the field of view; however the counterclockwise vortex generated during the upstroke is still present and can be seen under the wing. It has advected to the quarter chord location. This vortex pulls most of the streamwise momentum under the wing which in turn impedes the generation of a leading edge vortex on top of the wing.

The wake at the $50 \%$ spanwise location on the wing is characterized by the presence of clockwise vortices shed during the upstroke. Two of the vortices generated at the trailing edge are shed during the upstroke. The remaining wake vortices are due to the splitting of the leading edge vortex. One of the split vortices lies above wing plane, while the other remains parallel to it. The separation of the vortices has been observed in previous studies, and is reported to be due to the chain of inclined and interconnected vortex loops over oscillating wings ${ }^{26-28}$. As the wing plunges down, Figure 8 shows the trailing edge vortex shedding off the wing. A leading edge vortex develops on top of the wing before the midstroke, as seen in Figure 8.
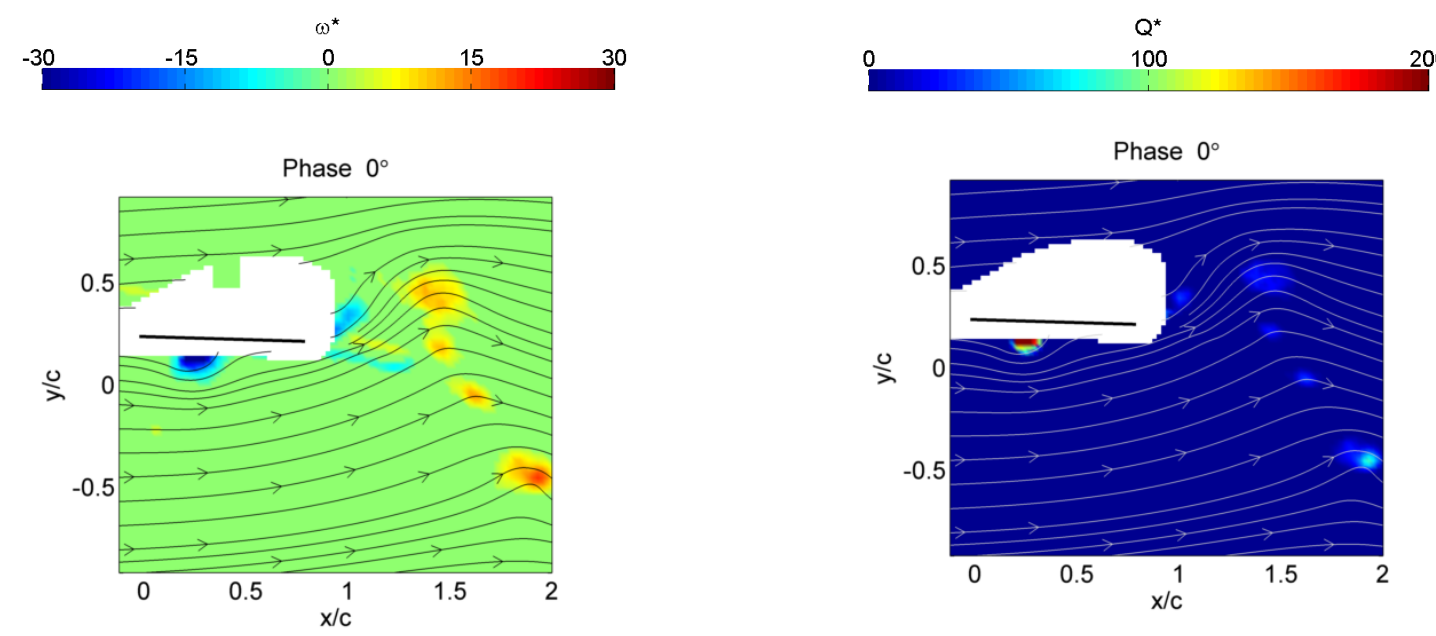

Figure 7. Vorticity and Q criterion at phase 0 degree for the $\mathrm{M} 1$ wing a $50 \%$ spanwise location. 

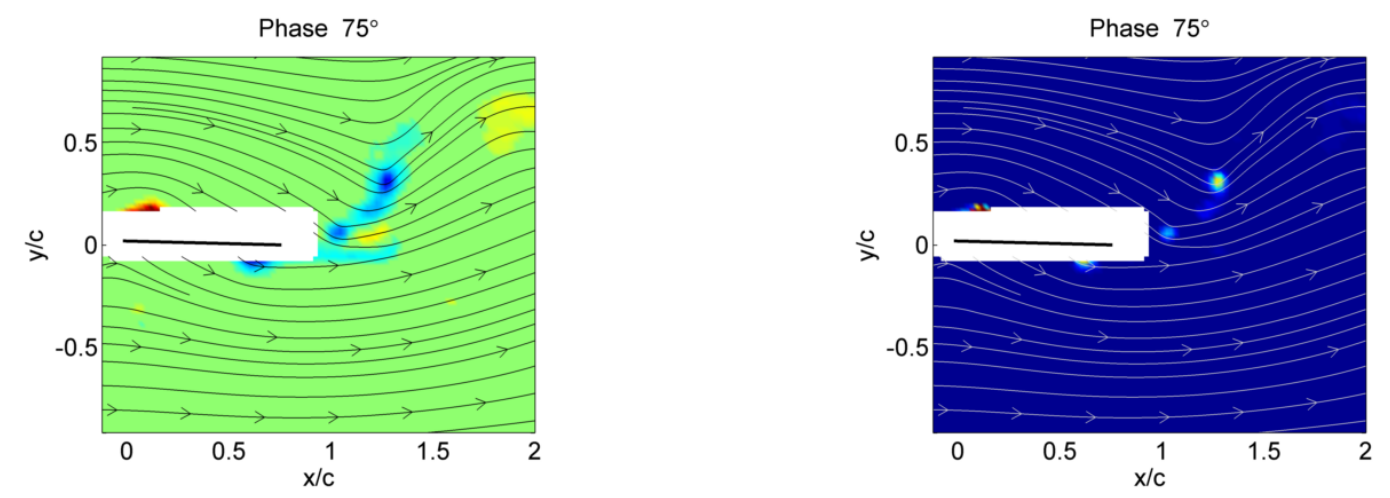

Figure 8. Vorticity and Q criterion at phase 75 degree for the $\mathrm{M} 1$ wing at a $\mathbf{5 0 \%}$ spanwise location.

Figures 9 and 10 present vorticity and Q-criterion contour at the $75 \%$ span locations for these for the 0 and 75 degree phases respectively. Figure 9 shows that the leading edge vortex created at the wing tip during upstroke is much large in size. This counter clockwise vortex is centered approximately at the $3 / 4$ position of the local chord length. Compared to the $50 \%$ span location, the vortex is further away from the wing. In the wake, one can see the trailing edge vortices shed during the downstroke. From this analysis, one can infer that these are slices of a vortex tube located in close proximity to the leading edge. This is similar to the trailing edge vortex tube which has already shed from the wing. The splitting of the leading edge vortex is not present at this phase in the motion unlike that at the beginning of the downstroke. This could be a consequence of the wing deflecting inwards towards the root of the wing. Figure 10 presents the flow field around the M1 wing at a phase of 75 degrees in the plunge motion and a spanwise location of $75 \%$. A leading edge vortex is seen to form on the upper surface of the wing. At the trailing edge, the LEV under the wing at the beginning of the downstroke has shed and correspondingly pulls the flow downwards at the aft of the wing. The vortices are seen to align, as the LEV pulls the flow towards the trailing edge of the wing, and since the previously shed vortex is now on top of the wing. Figure 11 shows the normalized velocity at the same phase and location as Figure 10. It can be seen that the flow is pulled down, thus creating a jetlike behavior between the vortices.
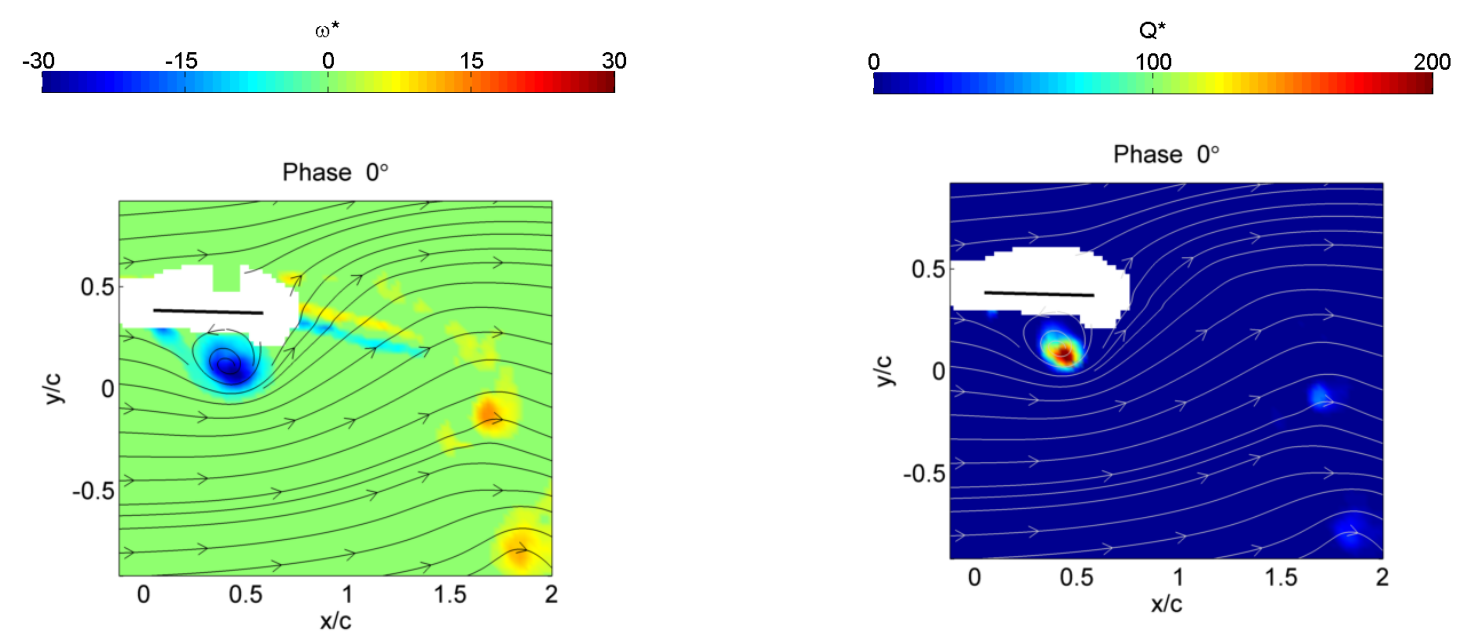

Figure 9. Vorticity and Q criterion at phase 0 degree for $M 1$ wing at the $75 \%$ spanwise plane. 

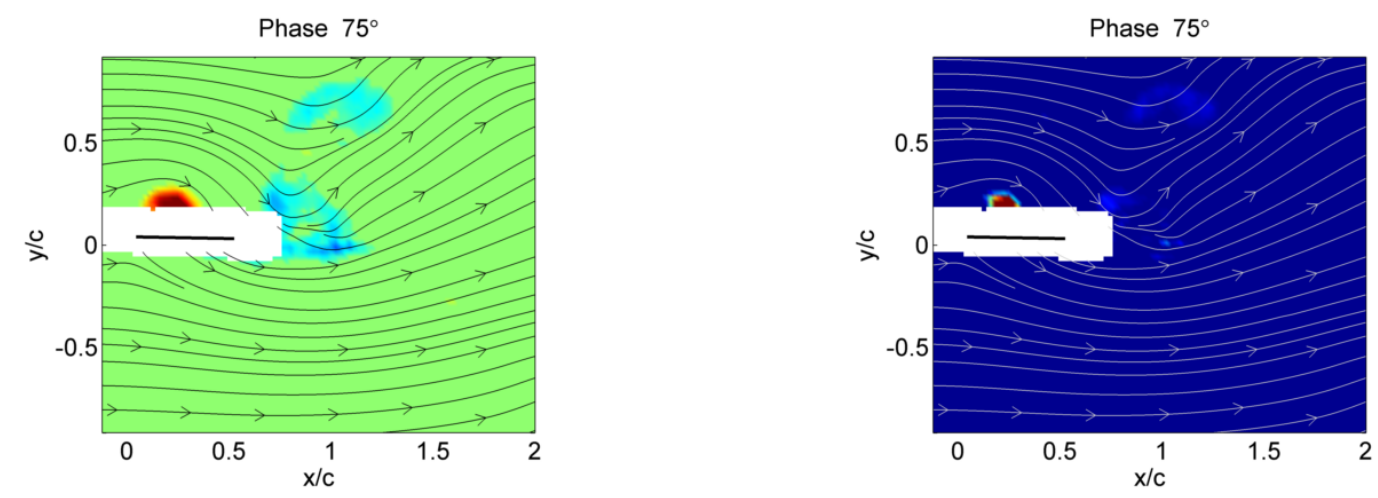

Figure 10. Vorticity and Q criterion at phase 75 degree for the $\mathrm{M} 1$ wing at the $\mathbf{7 5 \%}$ spanwise plane.

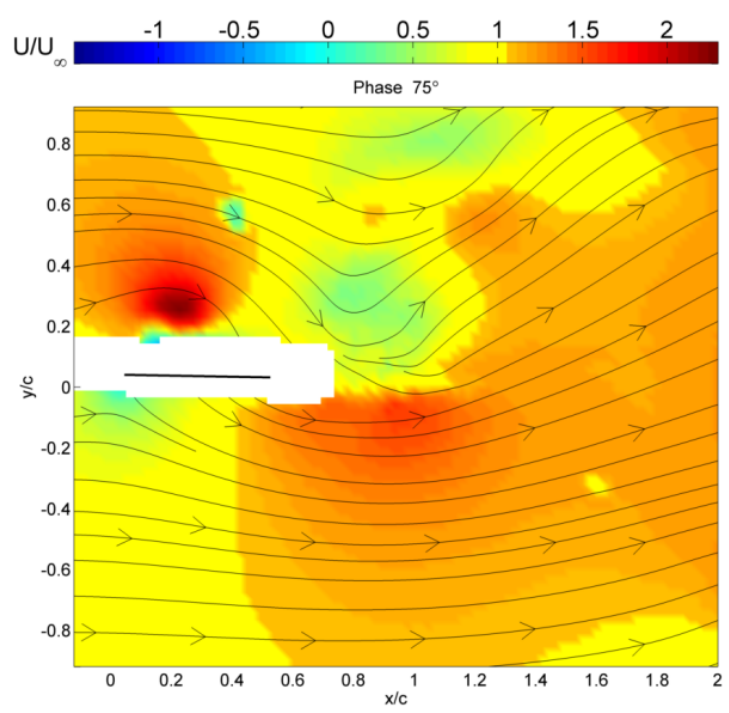

Figure 11. Normalized Velocity at phase 75 degree for M1 wing model at the $75 \%$ location.

\section{IV.B.2 M2 Model}

Figure 12 and Figure 13 present the vorticity and Q-criterion contours for the M2 wing throughout the downstroke at the spanwise locations of $50 \%$ and $75 \%$. Overall, the vortices are found to be much weaker for this wing than the M1 wing. The vorticity is constantly being shed at the trailing edge. From Figure 12 and 13, it is seen that the trailing edge vorticity rolls up into vortices. However, evidence of a coherent vortex tube existing throughout the downstroke is not found. This is a result of the wing displaying significant tip-root lag. This is representative of the midspan moving upward while the wing tip deflects downward. This behavior results in the creation of vortices of opposite sign. Figure 13 shows this behavior in the flow, where the flow at the midspan is pulled to the top of the wing, and at the $75 \%$ location the flow is pulled in the opposite direction. Since the wing is highly compliant there is no formation of a leading edge vortex, this also causes the wing to have a smaller tip deformation when compared to the M1 wing, as the root cannot drive the motion of the tip. 

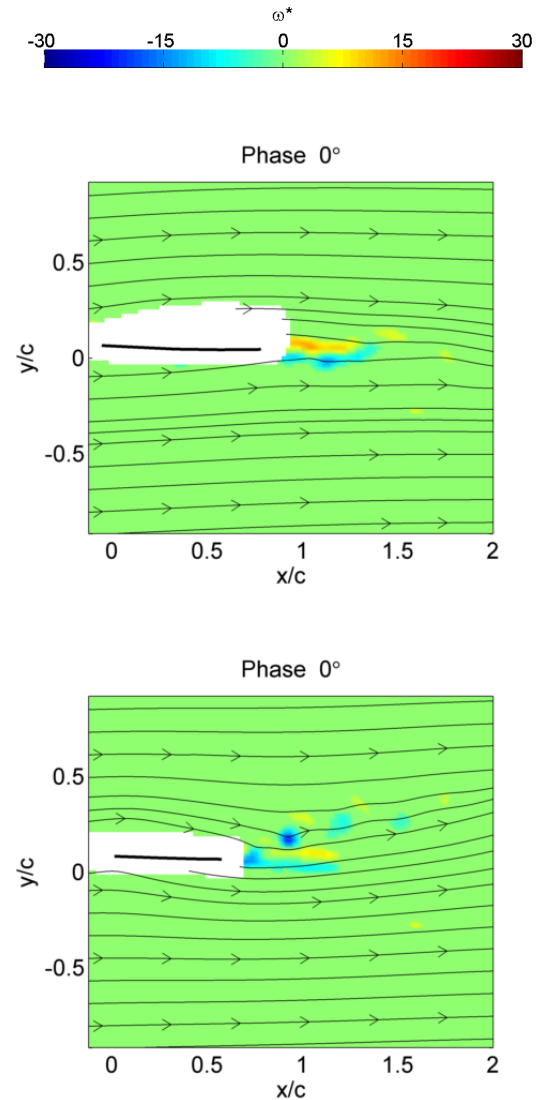
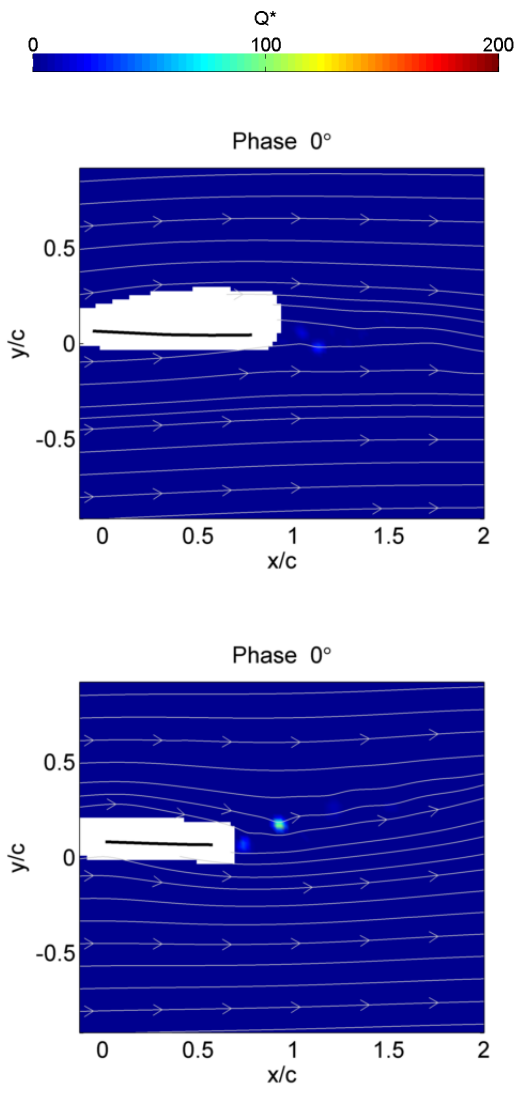

Figure 12. Vorticity and Q criterion at beginning of downstroke for M2 wing. Top: Midspan, Bottom: 75\% Span Location
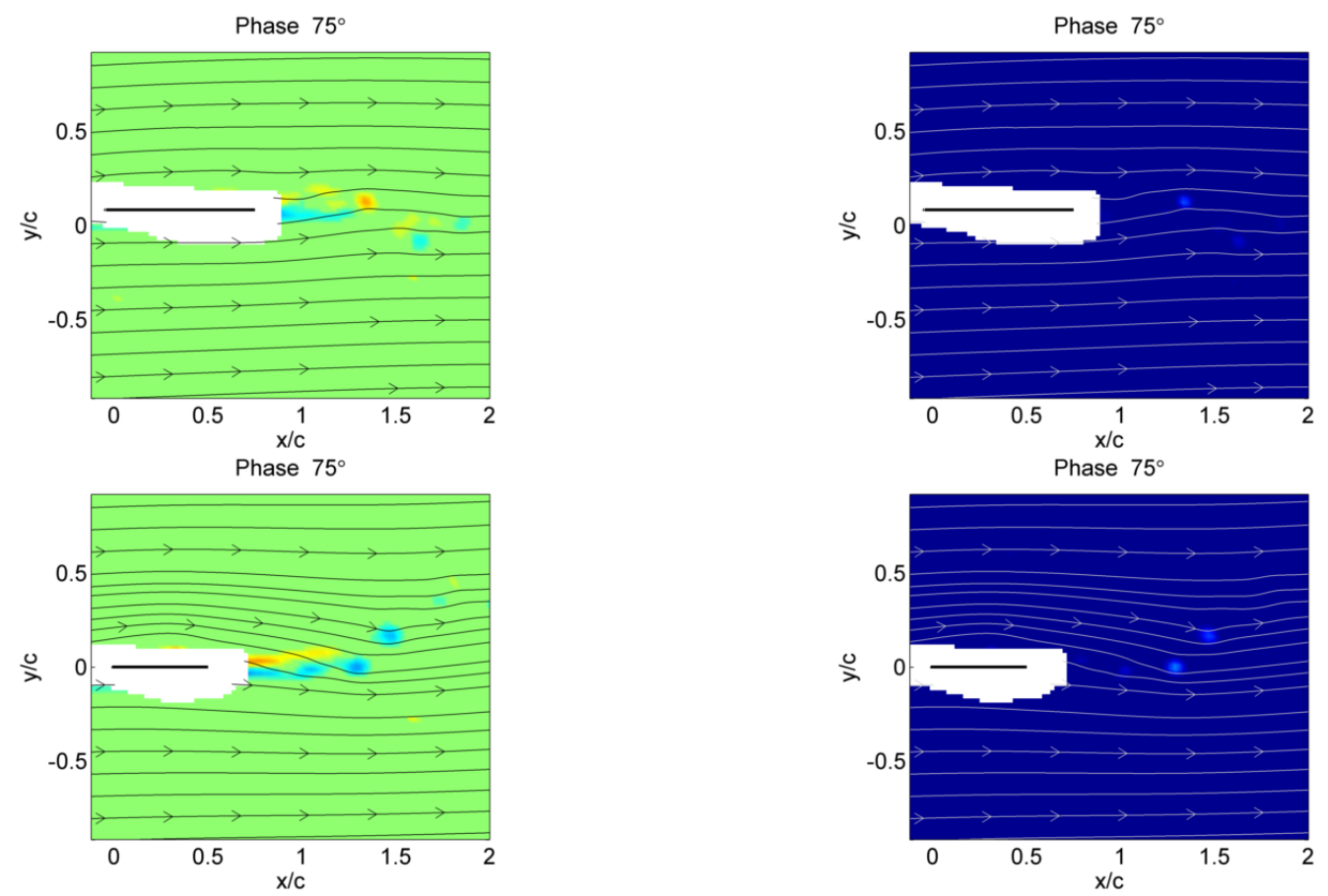

Figure 13. Vorticity and Q criterion near midstroke for M2 wing. Top: Midspan, Bottom: 75\% Span Location 


\section{IV.B.3 Comparison with water tunnel studies}

The water tunnel studies of Rausch et $\mathrm{al}^{3}$ showed insignificant wing deformation for wings with $\Pi_{1}$-parameter values greater than 1400 . The only model that displayed spanwise bending was the wing with the lowest value of $\Pi_{1}=30$. In that study the deformations are explained in terms of the reduced plunge amplitude of the motion $\left(\mathrm{h}_{\mathrm{o}}\right)$, where a positive percentage value signifies a motion greater than the root's motion, and a negative value, a reduction in the amplitude. The deformation was calculated to be $40 \%$ the amplitude of motion at the $75 \%$ location and less than $5 \%$ at the midspan. At the $75 \%$ span location, the deformation lagged the root by only $70^{\circ}$.

In contrast when performed in air, both wings showed significant spanwise deformation. The deformation for the M1wing $\left(\Pi_{1}=1702\right)$ was $32 \%$ and $120 \%$ of the amplitude of motion at $50 \%$ and $75 \%$ spanwise locations. Due to the inertia of the wing, the deformation at the $75 \%$ location is three times that of the flexible wing in water, even though the $\Pi_{1}$ parameter is two orders of magnitude greater. The M2 wing $\left(\Pi_{1}=55\right)$ showed the most unique behavior of all the 10 models in air. The deflections were not as large as the M1 wing because the wing was highly compliant. It showed large tip-root lag, with the tip going the opposite direction of the root for the majority of the motion. The deformations at the midspan were approximately $50 \%$ the amplitude of the motion. This results in the motion being only half the amplitude of input motion. The $75 \%$ spanwise location's displacement was approximately $2 \%$ less than the prescribed plunging motion of the root.

The PIV results from the water tunnel experiments show that the flow was dominated by the formation of a LEV during the downstroke for all the cases studied. Also, the LEV was shown to separate and advect toward the trailing edge for all the cases. In water, the HDPE model $\left(\Pi_{1}=1960\right)$ showed the formation of the LEV at the beginning of the downstroke at the $50 \%$ and $75 \%$ span locations. This vortex proceeded to advect, ultimately shedding from the trailing edge of the wing at the beginning of the next cycle $\left(30^{\circ}\right)$ at the $50 \%$ location. The LEV leaves the trailing edge at an earlier phase of $270^{\circ}$ for the $75 \%$ span location. The LEV for the model with a $\Pi_{1}=30$ began to form at a later phase than the rigid model. It was shown that the vortex at the $75 \%$ location is significantly larger than at the midspan. The vortex formed at phase of approximately $60^{\circ}$ for both spanwise locations. This coincides with the location of maximum spanwise deformation. The LEV was shown to shed from the trailing edge at a phase of $30^{\circ}$ in the next cycle of the motion for the $50 \%$ span plane. At the $75 \%$ plane, the LEV advects downstream from the TE at the beginning of the downstroke $\left(0^{\circ}\right)$. In air, the bending of the M1 wing blocked the camera during PIV, thus the formation of the LEV is not resolvable. The LEV at the $75 \%$ spanwise location is significantly larger than at the midspan, which is similar behavior to the LDPE wing in water. Figure 14 shows the vorticity at the phases where the LEV is seen to leave the trailing edge of the wing for the $50 \%$ and $75 \%$ locations. The LEV passes the trailing edge at a phase of $255^{\circ}$ at the midspan, and $45^{\circ}$ earlier at $75 \%$. The models in air show the LEV rising away from the trailing edge at earlier phases, than the similar models in water. At the $50 \%$ location, this occurs approximately $135^{\circ}$ earlier. At the $75 \%$ location it occurs $150^{\circ}$ earlier than the LDPE wing and $60^{\circ}$ earlier than the HDPE wing (same material as M1). The M2 wing in air did not show an LEV being formed.

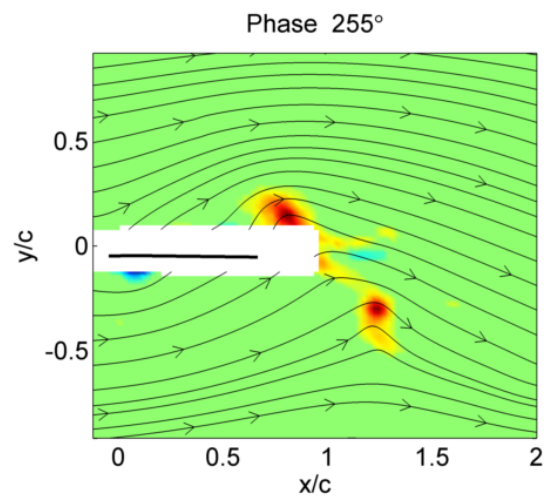

(a)

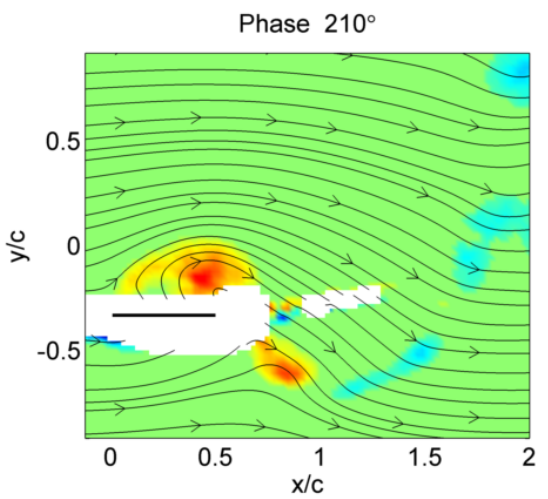

(b)

Figure 14. Vorticity plots for phases where the LEV leaves TE for M1 wing. (a) $50 \%$ span, (b) $75 \%$ span. 


\section{IV.C Force Calculations}

The force calculations are performed utilizing the control volume approach described in section III. The control volume spans from the $50 \%$ to the $90 \%$ spanwise locations and encompasses the entire field of view presented in the previous figures. The force results are presented in Figure 15 as a function of phase throughout the plunging cycle.

The M1 and M2 wings are represented by the blue and red lines in Figure 15 respectively. The maximum force in the vertical direction (Fy) for the M1 wing is seen to occur at the end of the downstroke, with a steep decrease after this phase. At the end of the downstroke, the leading edge vortex on top of the wing is still strong and has only been advected $40 \%$ of the chord. At the beginning of the upstroke, the vortex is located after the mid-chord. The lowest values of Fy occur halfway through the upstroke where the leading edge vortex created during the downstroke sheds from the trailing edge while a counter clockwise vortex is created on the underside of the wing. The vortex develops on the underside of the wing and creates a low pressure region on the bottom.

Horizontal Force (Fx) in the M1 model is characterized by the shedding of the vortices, as these align to create jet like behavior in the wake, as previously seen in Figure 11. The maximum Fx is achieved in the phase of the motion where the LEV is being shed. The production of this force starts when the bending is at its maximum and higher wing velocities are present. Similar results have been shown in previous experiments ${ }^{24}$ for a hovering wing; these results presented the maximum streamwise force occurred slightly before the maximum spanwise bending angle.

Fx and Fy for the M2 wing $\left(\Pi_{1}=55\right)$ are shown to be significantly smaller than the M1 wing. This is a result of a significant tip-root lag which has been shown to diminish the generation and development of unsteady vortex structures. Ultimately, insignificant amounts of horizontal and vertical forces are generated on the M2 wing.
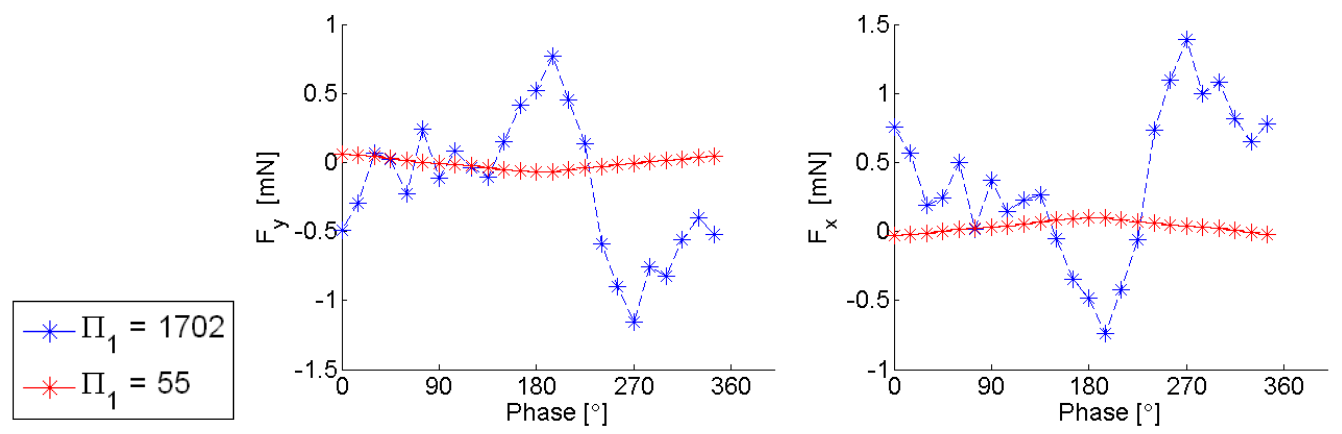

Figure 15. Phase Averaged Force Measurements for M1 and M2 wing.

\section{Summary}

A study of the effect of flexibility using the ratio of elastic to aerodynamic forces for forward flight conditions in air was performed. This study utilized Zimmerman planform wings and involved the examination of both the modal characteristics of the wing and the flow around the wing throughout the plunging cycle. The conditions tested allowed for examination of the effects of the wings stiffness parameter as well as a comparison to previous water tunnel experiments.

From the PIV measurements it was seen that both models ( $\Pi_{1}$ values of 55 and 1702) demonstrated significant deformations. This was caused by the high inertia of the plunging motion in air. Stronger leading edge vortices were formed for locations close to the tip, for the wing with a stiffness of 1702 showing a clear vortex tube throughout the spanwise locations. The M2 wing tested was highly flexible and showed significant tip-root lag, thus having weak vortices and being detrimental to force production. The M1wing showed similar behavior of that of a flexible flapping wing.

In contrast with the water tunnel experiments, results show significant deformation for both wings studied. This implies that wing loading and the ratio of structural density to fluid density are important when studying flexibility. However matching the kinematic scaling parameters and $\Pi_{1}$ alone does not ensure the fluid dynamic effects are present and one should also consider the effects of structural resonances of the wing. 


\title{
Acknowledgements
}

The authors would like the acknowledge support of the Air Force Office of Scientific Research under the MURI program in a joint effort with the University of Michigan. Additional support for this work has come from the Florida Center for Advanced Aero Propulsion. The authors would also like to acknowledge to the assistance of Erik Sällström for developing many of the data processing routines used in this study.

\section{References}

\begin{abstract}
${ }^{1}$ Shyy,W., Aono, H., Chimakurthi, S.K., Trizila, P., Kang, C.-K, Cesnik, C.E.S. and Liu, H., "Recent progress in flapping wing aerodynamics and aeroelasticity," Progress in Aerospace Sciences, vol. 46, no. 7, pp. 284-327, Oct. 2010.

${ }^{2}$ Tang, J., Viieru, D., and Shyy, W., "Effects of Reynolds Number and Flapping Kinematics on Hovering Aerodynamics," AIAA Journal, vol. 46, no. 4, pp. 967-976, Apr. 2008.

${ }^{3}$ Rausch, J.M., Baik,Y.S., Bernal,L.P., Cesnik, C. E. S., and Shyy,W., "Fluid Dynamics of Flapping Rigid and SpanwiseFlexible Elliptical Flat Plates at Low Reynolds Numbers," AIAA conf. paper:2010-4739, Proceedings of the 40th AIAA FDC, Chicago, July. 2010.

${ }^{4}$ Shyy,W., and Liu, H., "Flapping Wings and Aerodynamic Lift: The Role of Leading-Edge Vortices," AIAA Journal, vol. 45, no. 12, pp. 2817-2819, Dec. 2007.

${ }^{5}$ Baik,Y.S., Bernal, L. P., and Shyy, W., "Unsteady Force Generation and Vortex Dynamics of Pitching and Plunging Flat Plates at Low Reynolds Number," AIAA conf. paper: 2011-220, Proceedings of the 49th AIAA ASM, Orlando, Jan. 2011.

${ }^{6}$ Shyy,W., Lian, Y., Tang, J., Liu, H., Trizila, P., Stanford, B., Bernal, L., Cesnik, C., Friedmann, P., Ifju, P., "Computational aerodynamics of low Reynolds number plunging, pitching and flexible wings for MAV applications," Acta Mechanica Sinica,
\end{abstract} vol. 24, no. 4, pp. 351-373, Jul. 2008.

${ }^{7}$ Maxworthy,T., "The fluid dynamics of insect flight," Annual Review of Fluid Mechanics, vol. 13, pp. 329-350, 1981.

${ }^{8}$ Triantafyllou, M.S., Triantafyllou, G.S., and Yue, D.K.P., "Hydrodynamics of Fishlike Swimming," Annual Review of Fluid Mechanics, vol. 32, pp. 33-53, Jan. 2000.

${ }^{9}$ Heathcote, S., and Gursul, I., "Flexible Flapping Airfoil Propulsion at Low Reynolds Numbers," AIAA Journal, vol. 45, no. 5, pp. 1066-1079, May 2007.

${ }^{10}$ Heathcote, S., Wang, Z., and Gursul, I., "Effect of spanwise flexibility on flapping wing propulsion,” Journal of Fluids and Structures, vol. 24, no. 2, pp. 183-199, Feb. 2008.

${ }^{11}$ Taylor, G.K., Nudds, R.L., and Thomas, A.L.R., "Flying and swimming animals cruise at a Strouhal number tuned for high power efficiency," Nature, vol. 425, no. 6959, pp. 707-711, 2003.

${ }^{12}$ Kang, C.-kwon, Aono, H., and Cesnik, C.E.S., "Effects of Flexibility on the Aerodynamic Performance of Flapping Wings," AIAA conf. paper: 2011-2131, Proceedings of the 41st AIAA TFMC, Honolulu, June.2011.

${ }^{13}$ Thiria, B. and Godoy-Diana, R., "How wing compliance drives the efficiency of self-propelled flapping flyers," Physical Review E, vol. 82, no. 1, pp. 1-4, Jul. 2010.

${ }^{14}$ Kang, C.-kwon, Aono, H., Carlos. S, and Shyy, W., "A Scaling Parameter for the Thrust Generation of Flapping Flexible Wings," AIAA conf. paper: 2011-1313, Proceedings of the 49th AIAA ASM, Orlando, Jan.2011.

${ }^{15}$ Ramananarivo, S., Godoy-Diana, R., and Thiria, B., "Rather than resonance, flapping wing flyers may play on aerodynamics to improve performance.," Proceedings of the National Academy of Sciences of the United States of America, vol. 108, no. 15, pp. 5964-5969, Apr. 2011.

${ }^{16}$ Michelin, S. and Llewellyn Smith, S. G., "Resonance and propulsion performance of a heaving flexible wing," Physics of Fluids, vol. 21, no. 7, p. 071902, 2009.

${ }^{17}$ Masoud, H. and Alexeev, A., "Resonance of flexible flapping wings at low Reynolds number," Physical Review E, vol. 81, no. 5, pp. 1-5, May 2010.

${ }^{18}$ Vanella, M., Fitzgerald, T., Preidikman, S., Balaras, E., and Balachandran, B., "Influence of flexibility on the aerodynamic performance of a hovering wing.," The Journal of Experimental Biology, vol. 212, no. 1, pp. 95-105, Jan. 2009.

${ }^{19}$ Albertani, R., Khambatta, P., Hart, A., Ukeiley, L., Oyarzun, M., Cattafesta, L., Abate, G., "Validation of a Low Reynolds Number Aerodynamic Characterization Facility," AIAA conf. paper: 2009-880, Proceedings of the 47th AIAA ASM, Orlando, Jan. 2009.

${ }^{20}$ Baik, Y.S., Rausch, J.M., Bernal, L.P., Shyy, W., and Ol, M.V., "Experimental Study of Governing Parameters in Pitching and Plunging Airfoil at Low Reynolds Number," AIAA conf. paper: 2010-388, Proceedings of the 48th AIAA ASM, Orlando, Jan. 2010.

${ }^{21}$ Rausch, J.M., Bernal, L.P., Cesnik, C.S, Shyy, W., and Ukeiley, L., "Fluid Dynamic Forces on Plunging Spanwise-Flexible Elliptical Flat Plates at Low Reynolds Numbers," AIAA conf. paper: 2011-3435, Proceedings of the 41st AIAA FDC, Honolulu, Jun. 2011.

${ }^{22}$ Ishihara, D., Horie, T., and Denda, M., "A two-dimensional computational study on the fluid-structure interaction cause of wing pitch changes in dipteran flapping flight.," The Journal of Experimental Biology, vol. 212, pp. 1-10, Jan. 2009.

${ }^{23}$ Sallstrom, E., "Flow Field of Flexible Flapping Wings," PHD Thesis, University of Florida, Gainesville, FL, 2011.

${ }^{24}$ Sallstrom, E., Ukeiley, L., Wu, P., and Ifju, P., “Aerodynamic Forces on Flexible Flapping Wings," AIAA conf. paper: 2011-569, Proceedings of the 49th AIAA ASM, Orlando, Jan. 2011. 
${ }^{25}$ Haller, G., “An objective definition of a vortex,” Journal of Fluid Mechanics, vol. 525, pp. 1-26, Feb. 2005.

${ }^{26}$ Platzer, M.F., Jones, K.D., Young, J., and Lai, J.C.S., "Flapping-Wing Aerodynamics: Progress and Challenges," AIAA Journal, vol. 46, no. 9, pp. 2136-2149, Sep. 2008.

${ }^{27}$ Jones, K.D. and Platzer, M.F., "Bio-Inspired Design of Flapping Wing Micro Air Vehicles - An Engineer's Perspective," AIAA conf. paper: 2006-37, Proceedings of the 44th AIAA ASM, Reno, Jan. 2006.

${ }^{28}$ Bozkurttas, M., Dong, H., Mittal, R., Madden, P., and Lauder, G.V., "Hydrodynamic Performance of Deformable Fish Fins and Flapping Foils," AIAA conf. paper: 2006-1392, Proceedings of the 44th AIAA ASM, Reno, Jan. 2006. 